\title{
Isolation of Current Components and Partial Reaction Cycles in the Glial Glutamate Transporter EAAT2
}

\author{
Thomas S. Otis ${ }^{1}$ and Michael P. Kavanaugh ${ }^{2}$ \\ ${ }^{1}$ Department of Neurobiology, University of California, Los Angeles Medical Center, Los Angeles, California 90095-1763, \\ and ${ }^{2}$ Vollum Institute, Portland, Oregon 97202
}

The kinetic properties of the excitatory amino acid transporter EAAT2 were studied using rapid applications of L-glutamate to outside-out patches excised from transfected human embryonic kidney 293 cells. In the presence of the highly permeant anion $\mathrm{SCN}^{-}$, pulses of glutamate rapidly activated transient anion channel currents mediated by the transporter. In the presence of the impermeant anion gluconate, glutamate pulses activated smaller currents predicted to result from stoichiometric flux of cotransported ions. Both anion and stoichiometric currents displayed similar kinetics, suggesting that anion channel gating and stoichiometric charge movements are linked to

Clearance of neurotransmitter released by glutamatergic excitatory synapses is accomplished by the activity of sodium-dependent transporters located on glial and neuronal cell membranes. Five distinct members [excitatory amino acid transporter EAAT1 (GLAST), EAAT2 (GLT-1), EAAT3 (EAAC1), EAAT4, and EAAT5] of a gene family encoding glutamate (Glu) transporters have been identified to date (Kanai and Hediger, 1992; Pines et al., 1992; Storck et al., 1992; Fairman et al., 1995; Arriza et al., 1997). As expected, expression of each of the resulting gene products confers the ability to accumulate radiolabeled glutamate. Expression also gives rise to an anion conductance (Fairman et al., 1995; Wadiche et al., 1995a) consisting of a small anion leak in the absence of substrate and a larger anion conductance linked to substrate binding (Bergles and Jahr, 1997; Otis and Jahr, 1998; Wadiche and Kavanaugh, 1998). For this reason, electrophysiological measurements of transporter currents typically monitor the sum of two currents, a relatively large anion current, and a smaller current generated by stoichiometric movement of cotransported ions and substrate.

The present study uses rapid solution exchange techniques applied to outside-out patches containing EAAT2 transporters to determine the kinetic relationship between the two components of glutamate transporter current. Understanding the link between gating of the anion conductance and the transport cycle is important in several respects. Although anion currents can be readily detected, it is difficult to measure the smaller stoichiometric transporter currents in physiological contexts, such as in native

\footnotetext{
Received Oct. 25, 1999; revised Jan. 24, 2000; accepted Jan. 31, 2000.

This work was supported by National Institutes of Health Grant NS33270. We are grateful to D. Bergles, C. Jahr, and J. Wadiche, as well as A. Tzingounis for discussions during this work. We thank Dr. J Dunlop for the generous gift of the HEK 293 cell line.

Correspondence should be addressed to Thomas S. Otis, Department of Neurobiology, University of California, Los Angeles Medical Center, 650 Charles Young Drive, Box 951763, Los Angeles, CA 90095-1763. E-mail: otist@ucla.edu. Copyright (C) 2000 Society for Neuroscience $0270-6474 / 00 / 202749-09 \$ 15.00 / 0$
}

early transitions in the transport cycle. Transporter-mediated anion currents were recorded with ion and glutamate gradients favoring either unidirectional influx or exchange. Analysis of deactivation and recovery kinetics in these two conditions suggests that, after binding, translocation of substrate is more likely than unbinding under physiological conditions. The kinetic properties of EAAT2, the dominant glutamate transporter in brain astrocytes, distinguish it as an efficient sink for synaptically released glutamate.

Key words: GLT1; glutamate uptake; anion conductance; EAAT2; astrocyte; glutamate transporter cells engaged in excitatory synaptic transmission (Otis et al., 1997). To estimate parameters of glutamate transport at functioning synapses based on measurements of the larger anion currents, it is important to establish the relationship between transporter current and glutamate flux. Transport is a multistep process involving the binding of substrate and ions, translocation, and the unbinding of substrate and ions to the intracellular space. During this process, net charge is moved across the membrane field; this charge movement generates the stoichiometric current. Kinetic analysis of these currents is necessary to establish the precise timing of individual steps in the transport cycle, information critical for evaluating the role of transporters in buffering and sequestering synaptically released glutamate (Diamond and Jahr, 1997; Mennerick et al., 1999).

Our results on EAAT2 transporters identify a tight linkage between the two components of glutamate transporter current. Furthermore, they suggest that both currents arise from closely connected states occupied at early steps in the transport cycle, a proposal supported by an extremely simple mathematical model. Recordings with ion gradients designed to compare presteadystate kinetics of glutamate exchange with those of unidirectional influx suggest that, after binding, glutamate is rapidly translocated by EAAT2. Moreover, binding and translocation steps take place on a much faster time scale than does a complete transport cycle. Finally, recombinant EAAT2 shows very similar kinetics to transporter currents evoked in patches from hippocampal astrocytes (Bergles and Jahr, 1997), consistent with the proposal that EAAT2 (GLT1) is responsible for the majority of glutamate transport across astrocyte plasma membranes (Rothstein et al., 1996).

\section{MATERIALS AND METHODS}

Human embryonic kidney 293 cells. Human embryonic kidney HEK 293 (HEK 293) cells stably transfected with an ecdysone-inducible plasmid (Invitrogen, Carlsbad, CA) containing the human EAAT2 cDNA were a generous gift from Dr. John Dunlop (Wyeth-Ayerst, Princeton, NJ). 
Cells were cultured in DMEM (catalog \#10569-010; Life Technologies, Gaithersburg, MD) supplemented with $10 \%$ fetal bovine serum, 100 $\mathrm{U} / \mathrm{ml}$ penicillin-streptomycin (catalog \#15140-122; Life Technologies), $0.4 \mathrm{mg} / \mathrm{ml} \mathrm{G} 418$ (Geneticin, catalog \#10131-019; Life Technologies), and $0.2 \mathrm{mg} / \mathrm{ml} \mathrm{Zeocin} \mathrm{(catalog} \mathrm{\# 45-0430;} \mathrm{Invitrogen).} \mathrm{EAAT2} \mathrm{expres-}$ sion was induced by exposure of the cells to $5-10 \mu \mathrm{g} / \mathrm{ml}$ of the ecdysone analog ponasterone A (Sigma, St. Louis, MO) for 24-72 hr. Peak expression, assayed by uptake of radiolabeled glutamate, occurred 24-48 $\mathrm{hr}$ after addition of hormone to the culture medium.

Electrophysiology. All recordings were made with an Axopatch 200B amplifier (Axon Instruments, Foster City, CA) and at temperatures of $21-23{ }^{\circ} \mathrm{C}$. Signals were filtered at $2-5 \mathrm{kHz}$ and digitized at $10-20 \mathrm{kHz}$. In whole-cell recordings, pipette solutions consisted of (in mM): $135 \mathrm{CsNO}_{3}$, $10 \mathrm{HEPES}, 10$ tetraethylammonium (TEA)-Cl, 10 EGTA, and $1 \mathrm{MgCl}_{2}$, adjusted to $\mathrm{pH} 7.3$ with $\mathrm{CsOH}$.

Outside-out patch recordings and rapid solution exchange. Pipettes glass (World Precision Instruments, Sarasota, FL) was pulled to make electrodes with resistances of 1-3 M $\Omega$ in the bath solution. Three different pipette solutions were used. To record transporter-associated currents under conditions that maximized net transport of glutamate, pipettes contained (in mM): either $135 \mathrm{KSCN}$ or $135 \mathrm{~K}$-gluconate. To record transporter-associated currents under conditions that ensured homoexchange of glutamate, pipettes contained (in mM): $130 \mathrm{NaSCN}$ and 10 Na-glutamate. All pipette solutions also contained (in mM): 10 HEPES, 10 TEA-Cl, 10 EGTA, and $1 \mathrm{MgCl}_{2}$, adjusted to $\mathrm{pH} 7.3$ with either $\mathrm{KOH}$ or $\mathrm{NaOH}$ as appropriate.

Rapid solution exchange to outside-out patches was accomplished with a two-barreled application pipette attached to a piezoelectric bimorph. Each barrel of this application pipette was connected to a four- or six-way manifold allowing the application of multiple combinations of solutions to the same patch. At the end of each experiment, the recording pipette tip was cleared with positive pressure, and a solution of reduced ionic strength (diluted $50 \%$ ) was allowed to flow through the glutamatecontaining barrel. Jumps of the application pipette were then delivered, and the change in holding current was recorded. The resulting "open-tip" currents are displayed above each set of experimental traces and represent the approximate time course of solution exchange across the patch. A more complete description of these methods has been described previously (Otis and Jahr, 1998).

The control extracellular solution contained (in $\mathrm{mm}$ ): $135 \mathrm{NaCl}, 5.4$ $\mathrm{KCl}, 1.8 \mathrm{CaCl}_{2}, 1.3 \mathrm{MgCl}_{2}$, and 5 HEPES, adjusted to $\mathrm{pH} 7.4$ with $\mathrm{NaOH}$. For the current versus voltage analyses in different anion gradients, some of the patches were also exposed to (in mM): $135 \mathrm{NaSCN}, 5.4$ $\mathrm{KSCN}, 1.8 \mathrm{Ca}_{\text {gluconate }}, 1.3 \mathrm{Mg}$ gluconate $_{2}$, and $5 \mathrm{HEPES}$, adjusted to pH 7.4 with $\mathrm{NaOH}$. All chemicals were purchased from Sigma.

Data analysis. Data analysis was performed with pClamp 6.0 (Axon Instruments), Origin 5.0 (Microcal, Northampton, MA), and Igor (Wavemetrics, Lake Oswego, OR). Artifacts caused by the voltage pulse applied to the bimorph have been removed (Otis and Jahr, 1998). Error bars represent \pm 1 SEM. Student's $t$ test was used to determine confidence intervals.

Simulations. Simulations were performed using ScoP 3.51 (Simulation Resources Inc.; http://www.simresinc.com/menu.html).

\section{RESULTS}

\section{Whole-cell currents}

Whole-cell recordings were made from HEK 293 cells stably transfected with a ponasterone-inducible promoter driving expression of EAAT2 (see Materials and Methods). Pipettes containing $\mathrm{CsNO}_{3}$ solution were used to voltage-clamp cells, and whole-cell currents were recorded in response to application of $250 \mu \mathrm{M} \mathrm{L}-\mathrm{Glu}$. After induction, the cells exhibited large inward currents in response to Glu (Fig. 1A). Current-voltage $(I-V)$ relationships were measured by delivering a series of voltage steps [ -90 to $+50 \mathrm{mV} ; \Delta V$ of $10 \mathrm{mV}$; holding potential of $-50 \mathrm{mV}]$ in the absence and presence of Glu. Families of subtracted responses (Glu - control) are shown for a cell exposed to the inducing hormone (Fig. $1 A$ ) and for an untreated control cell (Fig. $1 B$ ). In Figure $1 C$, mean $I-V$ curves for three ponasteronetreated cells (filled circles) and for five untreated cells (open
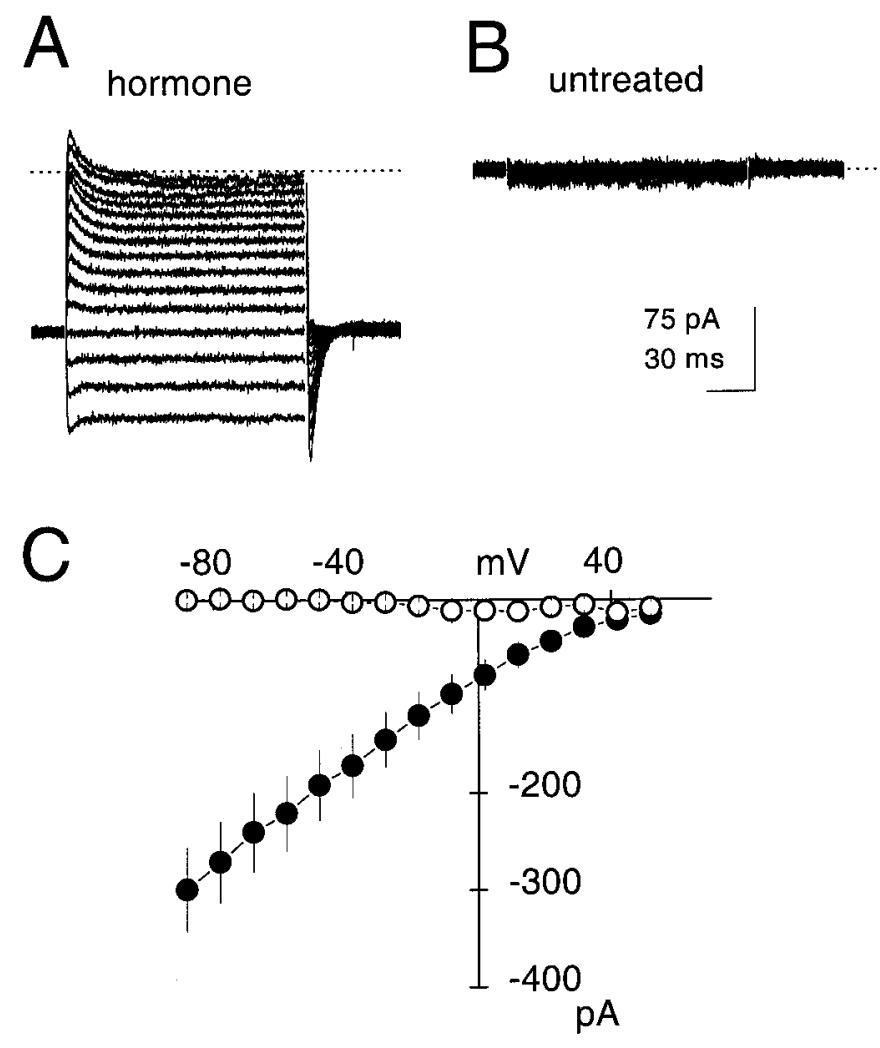

Figure 1. Whole-cell currents recorded in HEK 293 cells stably transfected with EAAT2 under the control of an inducible promoter. Application of the hormone ponasterone $\mathrm{A}$ for $>24 \mathrm{hr}$ causes the appearance of transporter currents activated by $250 \mu \mathrm{M}$ Glu. $A$, A family of voltage steps $(-90$ to $+50 \mathrm{mV} ; \Delta V$ of $10 \mathrm{mV}$; holding potential of $-50 \mathrm{mV}$ ) imposed on a hormone-treated cell in the presence of Glu. Currents recorded in the absence of Glu have been subtracted. $B$, A transfected cell that has not been exposed to hormone but was subjected to the same voltage-clamp protocol as in $A$. $C$, Mean $I-V$ relationships for untreated transfected cells $(\bigcirc ; n=5)$ and for cells treated with ponasterone $\mathrm{A}(\boldsymbol{\bullet}$ $n=4)$. Pipettes contained $\mathrm{CsNO}_{3}$-based solutions.

circles) show that hormone treated cells develop a glutamatedependent conductance that is inward at all membrane potentials tested, as expected given a high permeability to $\mathrm{NO}_{3}$ anions (Wadiche et al., 1995a; Levy et al., 1998; Wadiche and Kavanaugh, 1998). By comparison, there was no measurable conductance activated by glutamate in the untreated cells, demonstrating that, if HEK cells have endogenously expressed glutamate transporters, they are undetectable with these methods.

\section{Currents in outside-out patches}

The large whole-cell currents elicited by Glu indicated that it might be possible to record transporter responses in cell-free patches. To measure rapid dynamics of EAAT2 glutamate transporters in response to Glu concentration jumps, patches in the outside-out configuration were removed from cells that had been stimulated with ponasterone A for 24-72 hr. At negative holding potentials, patch currents evoked by a jump into $10 \mathrm{~mm}$ Glu showed rapid inward transients, followed by sustained current components (Fig. 2A). Outward current shifts were elicited by jumps from control solution into solution containing $500 \mu \mathrm{M}$ kainate, an EAAT2-selective competitive antagonist (Fig. 2B). The time constants of activation and deactivation of the outward current evoked by kainate were $1.17 \pm 0.1(n=5)$ and $6.2 \pm 0.4$ 


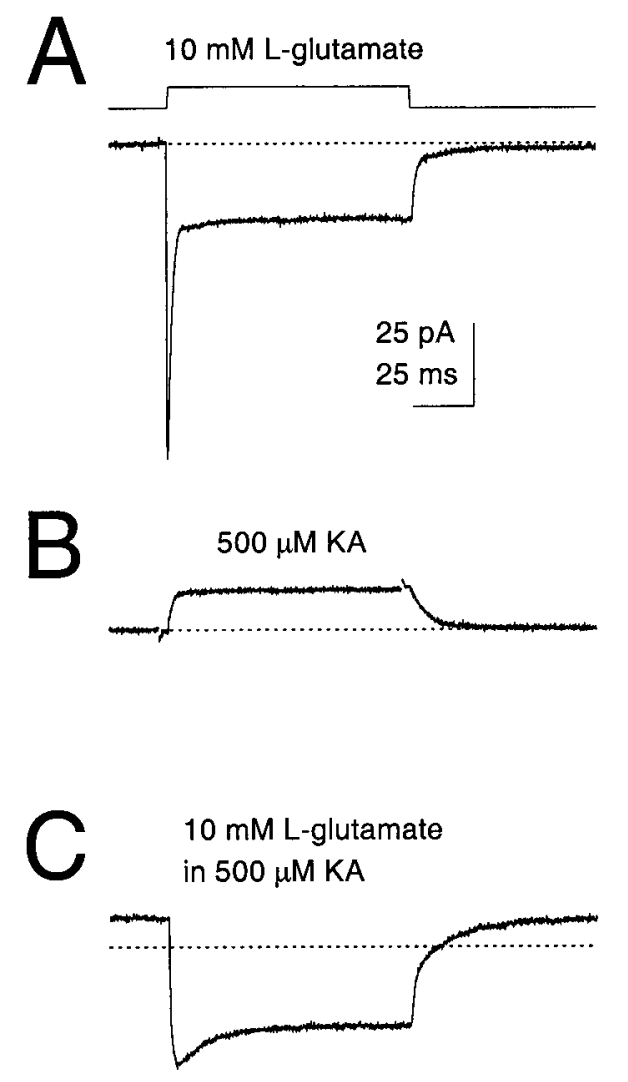

Figure 2. Transporter currents in an outside-out patch in response to agonist and antagonist concentration jumps. $A$, Transporter anion current in response to a $100 \mathrm{msec}$ duration, $10 \mathrm{~mm}$ pulse of Glu. The time course of the concentration jump in this and all subsequent figures is displayed directly above the response. $B$, A pulse of the EAAT2 antagonist kainate (100 msec, $500 \mu \mathrm{M}$ ) elicits an outward current because of the blockade of a persistent inward anion current observed in the absence of substrate. $C$, The response to $10 \mathrm{~mm}$ Glu in the continuous presence of $500 \mu \mathrm{M}$ kainate. Note the outward shift in the current trace (dotted trace indicates the current level before kainate application). The strong antagonism and slowing of the transient component is expected for a competitive antagonist that is not transported. Holding potential $\left(V_{\mathrm{h}}\right),-88 \mathrm{mV}$.

$(n=5)$ msec, respectively. Consider the two-state reaction, the simplest mechanism by which kainate may block the leak current:

$$
\text { kainate }+ \text { transporter } \underset{k_{\text {of }}}{\stackrel{k_{\text {on }}}{\rightleftharpoons}} \text { kainate }- \text { transporter }
$$

The experimentally measured time constants predict $k_{\text {on }}$ to be $1.7 \times 10^{6} \mathrm{M} / \mathrm{sec}^{-1}$, and $k_{\text {off }}$ equal to $161 \mathrm{sec}^{-1}$. These rate constants yield an affinity constant of $95 \mu \mathrm{M}$, in line with previous estimates (15-60 $\mu \mathrm{M})$ of kainate affinity (Arriza et al., 1994; Wadiche et al., 1995b).

In the continuous presence of $500 \mu \mathrm{M}$ kainate, responses to Glu jumps showed blunted peaks and larger steady-state components measured from the prejump baseline (Fig. 2C). These responses also showed a maintained outward shift in current caused by kainate, visible in Figure $2 B$ as the shift of baseline current level from the prekainate level indicated by the dotted line. Average results for kainate application to six patches are presented in Table 1, along with kinetic data measured from other patches exposed only to Glu. In general, EAAT2 responses appear qualitatively similar to those reported previously for other native and recombinant glutamate transporters in that (1) Glu jumps elicit a transient followed by a smaller steady-state component and (2) kainate or its analog dihydrokainate induces an apparent antagonism of a leak current (Bergles and Jahr, 1997; Otis and Jahr, 1998; Wadiche and Kavanaugh, 1998). The pharmacology and kinetics of EAAT2 were very similar to those reported for glutamate transporters present in patches from hippocampal astrocytes (Bergles and Jahr, 1997, 1998). Table 1 indicates that patch data from hippocampal astrocytes and from EAAT2 are statistically indistinguishable. The comparison suggests that the glutamate transporter currents described previously in CA1 astrocytes result either from GLT1 (EAAT2) or a functionally identical isoform.

\section{Patch currents under conditions favoring either transport or "exchange mode"}

Glutamate transporters are powered by transmembrane gradients of $\mathrm{Na}^{+}, \mathrm{K}^{+}, \mathrm{H}^{+}$, and substrate (Kanner and Sharon, 1978; Stallcup et al., 1979; Erecinska et al., 1983; Zerangue and Kavanaugh 1996b); therefore, changes in internal $\left[\mathrm{Na}^{+}\right]$and [Glu] are expected to alter transporter currents (Zerangue and Kavanaugh, 1996a; Levy et al., 1998). Figure 3 shows transporter current elicited by a jump into $10 \mathrm{~mm}$ Glu in two different patches, one with no internal $\left[\mathrm{Na}^{+}\right]$or [Glu] (Fig. $3 A$ ) and another with a pipette solution containing $140 \mathrm{~mm} \mathrm{Na}^{+}$and $10 \mathrm{~mm}$ Glu substituted for $\mathrm{K}^{+}$(Fig. 3B). On average, high $\left[\mathrm{Na}^{+}\right.$] and [Glu] in the pipette caused Glu-elicited currents to have a significantly larger steady-state component compared with control (control $I_{\text {ss }} / I_{\text {peak }}$, $0.2 \pm 0.02 ; n=14$; high $\left.\mathrm{Na}^{+}, 0.68 \pm 0.06 ; n=5 ; p<0.0005\right)$. This internal solution also converted the fast double-exponential decay observed at the end of the Glu pulse in control into a slower, single-exponential decay (control, $\tau_{1}$ of $1.3 \pm .11 \mathrm{msec} ; \tau_{2}$ of $18.7 \pm 2 \mathrm{msec} ; 71 \pm 3 \%$ fast; $n=12$; high $\mathrm{Na}^{+}, 21.6 \pm 2.7$ msec; $n=5$ ). This change in kinetics is consistent with the idea that a complete cycle of transport is highly unfavorable because of the high internal $\left[\mathrm{Na}^{+}\right]$and $[\mathrm{Glu}]$ and because of the absence of internal $\mathrm{K}^{+}$. With these ionic gradients, it is believed that the transporter operates as an exchanger, engaging in a futile shuttle in which the transporter brings Glu and coupled ions in and is forced by the high internal $\left[\mathrm{Na}^{+}\right]$and $[\mathrm{Glu}]$ to reverse and carry Glu and ions out. Indeed, measurements of substrate and ion fluxes support the idea no net $\mathrm{Na}^{+}$or Glu flux is accomplished under these conditions (Kanner and Sharon, 1978; Kanner and Bendahan, 1982; Kavanaugh et al., 1997).

The differences in decay kinetics after removal of external Glu (Fig. 3) may reflect two different fates of bound Glu. When operating as an exchanger, the current decay is expected to predominantly reflect rates of transition leading to Glu unbinding to the extracellular space, whereas with 0 internal $\left[\mathrm{Na}^{+}\right]$and [Glu], the decay can reflect an additional path: forward movement through a complete transport cycle. To explore this hypothesis, the voltage dependence of current decay was examined under conditions of 0 internal $\mathrm{Na}^{+}$and high internal $\mathrm{Na}^{+}$and Glu. A family of responses to $10 \mathrm{~mm}$ Glu jumps recorded at different membrane potentials ( -100 to $+40 ; \Delta V$ of $10 \mathrm{mV}$ ) are displayed for two such patches (Fig. $4 A, B$ ). Scaling the steady-state components for the two patches shows that, when operating with high internal $\mathrm{Na}^{+}$(Fig. 4B2), current decay is markedly voltagedependent, becoming faster at positive potentials. In contrast, internal solutions favoring net uptake $\left(0\right.$ internal $\left.\mathrm{Na}^{+}\right)$yield currents with little change in the decay rate over the same range of membrane voltages (Fig. $4 A_{2}$ ). Mean times to $1 / 2$-decay after 
Table 1. Kinetic and pharmacological parameters of patch currents from EAAT2 and hippocampal astrocytes

\begin{tabular}{llccccc} 
& $\begin{array}{l}\text { Peak of } \\
\text { transient }(\mathrm{pA})\end{array}$ & $\begin{array}{c}1 / 2 \text {-Decay time of } \\
\text { transient }(\mathrm{msec})\end{array}$ & $\begin{array}{c}1 / 2 \text {-Decay time } \\
\text { of offset (msec) }\end{array}$ & $\begin{array}{l}\text { Recovery time } \\
\text { constant (msec) }\end{array}$ & $I_{\text {steady state }} / I_{\text {peak }}$ & $I_{\text {kainate }} / I_{\text {control }}$ \\
\hline EAAT2 & $-200 \pm 59(14)$ & $1.07 \pm 0.06(14)$ & $\begin{array}{c}1.3 \pm 0.2(6) \\
*\end{array}$ & $18.4 \pm 2.8(9)$ & $0.2 \pm 0.02(14)$ & $0.38 \pm 0.02(6)$ \\
$\begin{array}{c}\text { Bergles and Jahr } \\
(1997,1998)\end{array}$ & $-55.2 \pm 1.6(32)$ & $1.35 \pm 0.09(8)$ & $2.91 \pm 0.17(8)$ & $23.7 \pm 0.5(4)$ & $0.16 \pm 0.01(30)$ & N.D. \\
\hline
\end{tabular}

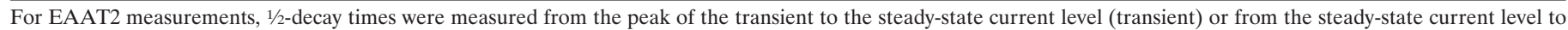

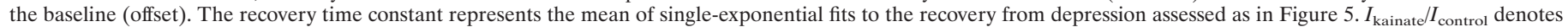

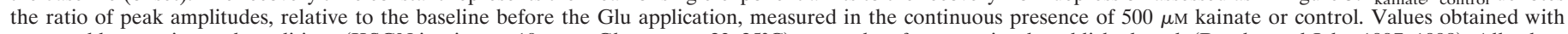

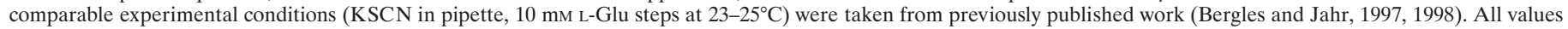
are expressed as mean \pm SEM (number of observations). The * indicates a significant difference at a level of $p \leq 0.01$. ND, Not determined.

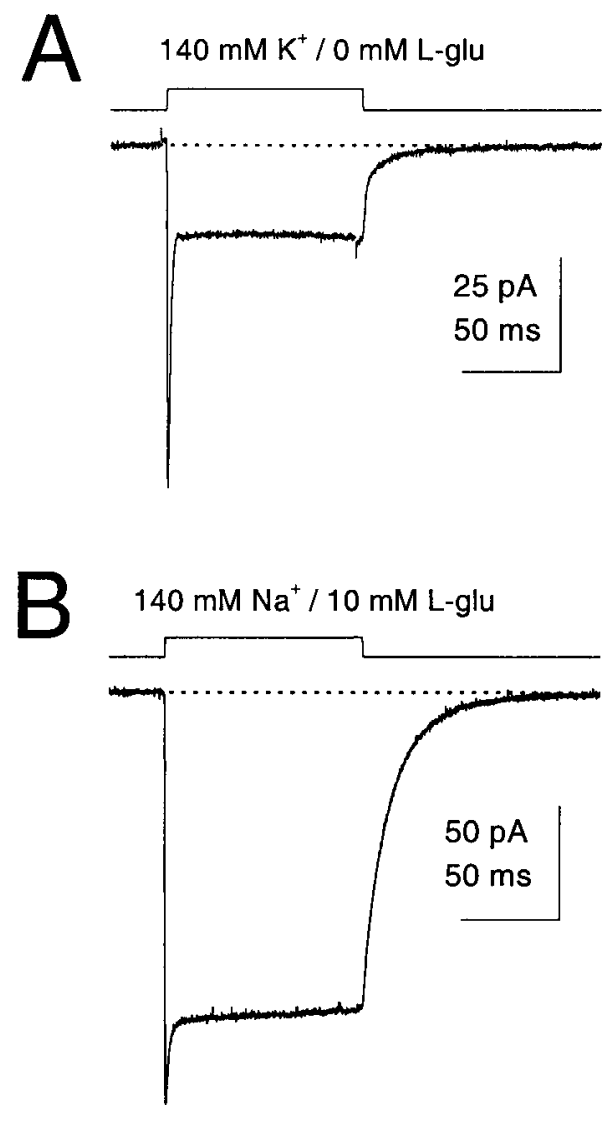

Figure 3. The kinetics of the transporter anion current are altered by high $\left[\mathrm{Na}^{+}\right]_{\text {in }}$ and $[\mathrm{Glu}]_{\text {in }} . A, B$, Responses in two different outside-out patches to a $100 \mathrm{msec}$ pulse of $10 \mathrm{~mm}$ Glu. In $A$, the pipette contained 140 $\mathrm{mm}$ KSCN and in $B, 130 \mathrm{~mm}$ NaSCN plus $10 \mathrm{~mm} \mathrm{NaGlu.} V_{\mathrm{h}},-87 \mathrm{mV}$ for both patches.

the offset of the Glu step were measured as a function of voltage and are displayed for comparison in Figure $4 C$. A simple explanation for these results is that, upon removal of $10 \mathrm{~mm}$ Glu, the transporter current deactivates by different routes in the two experimental conditions. In the high internal $\mathrm{Na}^{+} / \mathrm{Glu}$ condition, upon removal of external Glu, relaxation to steady state occurs as transporters reverse and Glu dissociates into the extracellular space. However, with 0 internal $\mathrm{Na}^{+}$, complete forward cycles of transport occur. In this situation, transporters rarely reverse and allow Glu to unbind to the outside. The sign of the voltage dependence when the transporters are operating as exchangers (faster deactivation at positive potentials) suggests that net positive charge is being expelled from the cell during deactivation.
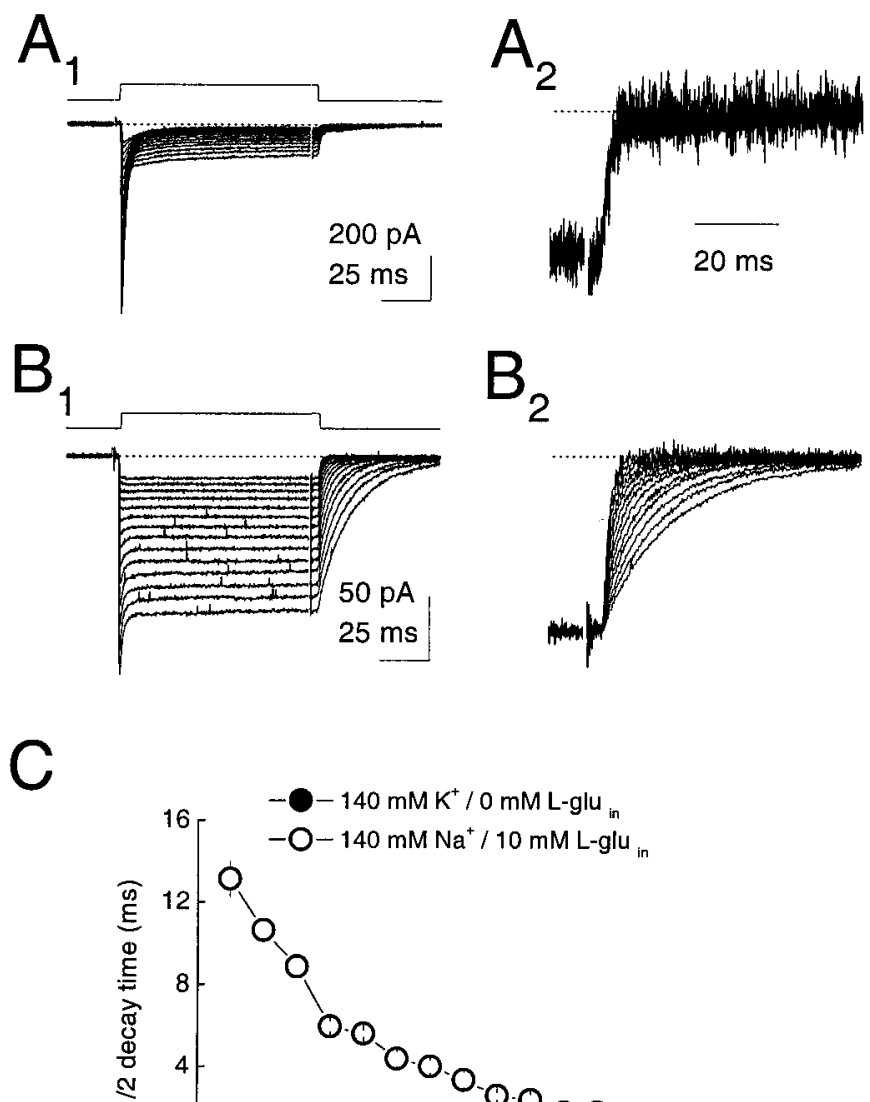

$\mathrm{B}_{2}$
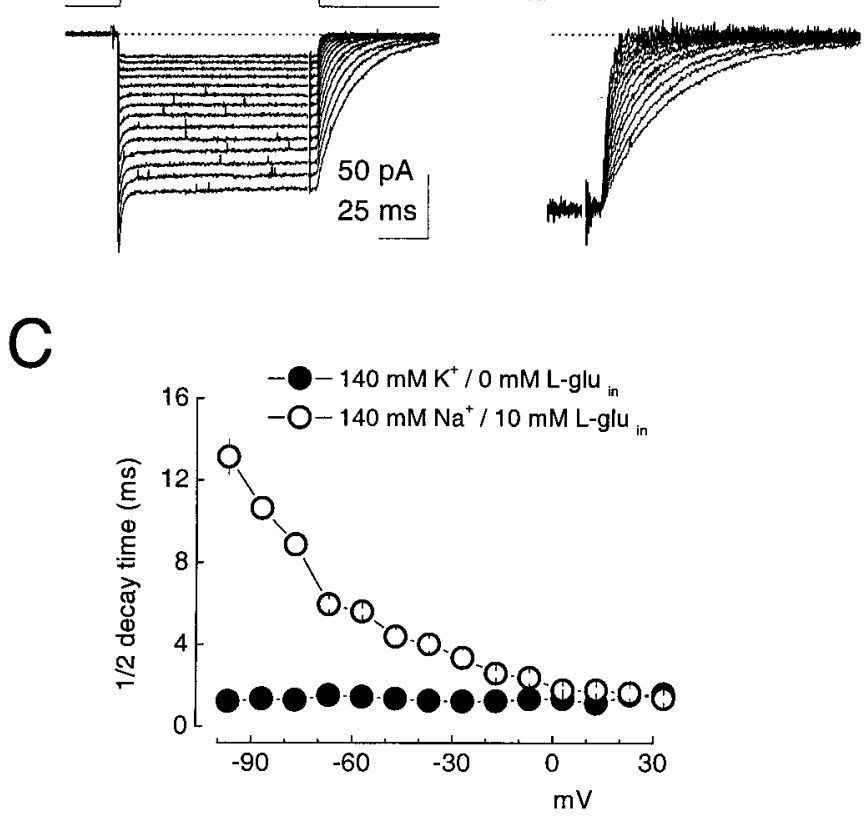

Figure 4. The voltage dependence of the decay after the end of the Glu pulse depends on the pipette solution. $A_{1}$, A family of transporter currents elicited with $10 \mathrm{~mm}$ Glu steps recorded at different membrane potentials $(-98$ to $+32 \mathrm{mV} ; \Delta V, 10 \mathrm{mV})$. A KSCN solution was in the pipette. $\mathrm{A}_{2}$ shows the normalized decays at the end of the Glu pulse. $\mathrm{B}_{1}$, A similar voltage-clamp protocol $(-97$ to $+33 \mathrm{mV} ; \Delta V, 10 \mathrm{mV})$ applied to a different patch recorded with $140 \mathrm{mM} \mathrm{Na}^{+}$and $10 \mathrm{~mm}$ Glu in the pipette. $\mathrm{B}_{2}$, Normalized decay phases. C, Mean $1 / 2$-decay times as a function of membrane potential for patches recorded with pipette solutions containing $140 \mathrm{~mm} \mathrm{KSCN}(\bullet ; n=14)$ or $140 \mathrm{mM} \mathrm{Na}^{+}$and $10 \mathrm{~mm} \mathrm{Glu}(\bigcirc ; n=$ 5). Note the lack of voltage dependence to the decay with high $\left[\mathrm{K}^{+}\right]$and the marked voltage dependence with high $\left[\mathrm{Na}^{+}\right]$and $[\mathrm{Glu}]$ in the pipette.

This is the opposite of the voltage dependence of normal uptake (slower forward rates at positive potentials) and is consistent with reversal of the transporter and movement of the positively charged $\mathrm{Na}^{+}$ions outward through the membrane voltage field. 

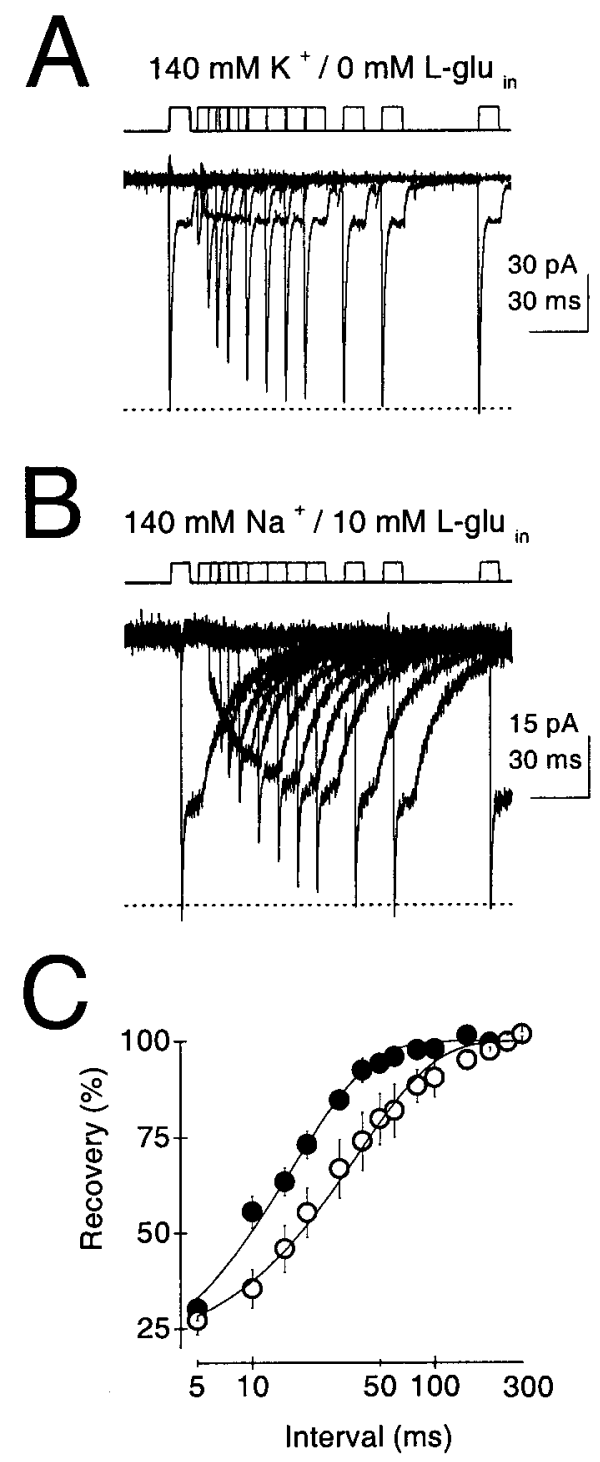

Figure 5. The rate of recovery from depression of the transporter current depends on the pipette solution. $A, B$, Responses to pairs of $10 \mathrm{msec}$ steps into $10 \mathrm{~mm}$ Glu with the indicated pipette solutions. Intervals of 5, 10, 15, $20,30,40,50,60,80,100$, and $150 \mathrm{msec}$ are displayed. A response to a single pulse of Glu has been subtracted from each displayed response. The dotted trace indicates the mean peak current of the response to a single pulse. $V_{\mathrm{h}},-88 \mathrm{mV}$ in $A$ and $-101 \mathrm{mV}$ in $B . C$, Mean amount of recovery (peak of the 2 nd response/peak of the 1st) plotted against the interval between pulses (note the log scale). indicates $140 \mathrm{~mm}[\mathrm{KSCN}]_{\text {in }}(n=9)$, and $\bigcirc$ indicates $140 \mathrm{~mm}\left[\mathrm{Na}^{+}\right]_{\text {in }}$ and $10 \mathrm{~mm}[\mathrm{Glu}](n=5)$. Lines display the means of single-exponential fits to the individual patches with $\tau$ values of 18.4 and $38.7 \mathrm{msec}$ for the $\mathrm{K}^{+}$and high $\mathrm{Na}^{+}$solutions, respectively.

Under 0 internal $\mathrm{Na}^{+}$conditions, if most transporters successfully complete cycles, then the average time required for a single cycle of transport will dictate the rate of recovery from the steady-state current level (Otis and Jahr, 1998). On the other hand, under exchange conditions, rates of reversal and/or unbinding will set the recovery time course. Figure 5 compares recovery time courses with 0 internal $\mathrm{Na}^{+}$and high internal $\mathrm{Na}^{+} / \mathrm{Glu}$ by delivering pairs of pulses of Glu separated by various intervals. As is evident from the single patches (Fig. $5 A, B$ ) and from the group data (Fig. $5 C$ ), recovery is slower with high $\mathrm{Na}^{+} / \mathrm{Glu}$ pipette solutions. Average single-exponential fits to recovery data from individual patches with 0 internal $\mathrm{Na}^{+}(18.4 \pm 2.8 \mathrm{msec}$; $n=9)$ and with high internal $\mathrm{Na}^{+} / \mathrm{Glu}(38.7 \pm 10.3 \mathrm{msec} ; n=5)$ are superimposed on the data in Figure $5 C$. Interestingly, under exchange conditions, the rate of decay upon Glu removal and the rate of recovery are more similar $(21.6$ and $38.7 \mathrm{msec}$, respectively) than the same rates with 0 internal $\mathrm{Na}^{+}$(1.3 and 18.4 msec, respectively). This can be explained as follows: with high internal $\mathrm{Na}^{+} / \mathrm{Glu}$, the anion current deactivates and recovers by the same route (unbinding to the outside), but with 0 internal $\mathrm{Na}^{+}$, anion current deactivates by forward movement into nonconducting states further along in the cycle (a fast process) and recovers only upon completion of an entire cycle (a slower process).

\section{Different anion gradients isolate the stoichiometric and anion current components}

The conditions used to record currents in the previous experiments $\left(140 \mathrm{~mm} \mathrm{SCN}^{-}\right.$in the pipette) cause the anion current component to dominate the total transporter current. Many of the interpretations in this study are based on the premise that the anion current is tightly linked to the transport cycle and that conducting states are accessible only from limited regions of that cycle. This premise predicts that the kinetics of the two components of transporter current should be correlated.

To isolate the different components of transporter current, $I-V$ relationships in response to $10 \mathrm{~mm}$ Glu jumps were measured using four different anion gradients: $\mathrm{SCN}^{-}{ }_{\text {in }} / \mathrm{Cl}^{-}{ }_{\text {out }}, \mathrm{SCN}^{-}{ }_{\text {in }} /$ $\mathrm{SCN}^{-}$out, gluconate ${ }^{-}{ }_{\text {in }} / \mathrm{SCN}^{-}$out, and gluconate ${ }^{-}{ }_{\text {in }} / \mathrm{Cl}^{-}$out in which the indicated anion is present at a concentration of 135-150 mM. Previous estimates of permeability have established that $\mathrm{SCN}^{-}$is $\sim 70$ times more permeable than $\mathrm{Cl}^{-}$and that gluconate $^{-}$is impermeable (Wadiche and Kavanaugh, 1998). Figure $6 A_{1}-D_{1}$ displays families of superimposed responses recorded at different membrane potentials $(-100$ to $+50 \mathrm{mV} ; \Delta V$ of $10 \mathrm{mV})$. These traces were recorded from three different patches $\left(C_{1}\right.$ and $D_{1}$ are from the same patch) exposed to the anion gradients listed above. Mean $I-V$ relationships are shown to the right. As expected, the reversal potentials ( $E_{\text {rev }}$ values) shift with changes in the anion gradient. With an $\mathrm{SCN}^{-}{ }_{\text {in }} / \mathrm{Cl}^{-}$out gradient, $E_{\mathrm{rev}}$ was more than $+40 \mathrm{mV}(n=11)$ and thus not determinable; with $\mathrm{SCN}^{-}{ }_{\text {in }} / \mathrm{SCN}^{-}$out, $E_{\text {rev }}$ was $+3 \pm 2 \mathrm{mV}(n=4)$; with gluconate $^{-}{ }_{\text {in }} / \mathrm{SCN}^{-}{ }_{\text {out }}, E_{\text {rev }}$ was $-59 \pm 2 \mathrm{mV}(n=8)$; and with gluconate ${ }^{-}{ }_{\text {in }} / \mathrm{Cl}^{-}$out,$E_{\text {rev }}$ was more than $+50 \mathrm{mV}$ and not measurable $(n=7)$. How do responses dominated by anion current (Fig. 6A) compare in size with responses dominated by stoichiometric current (Fig. 6D)? Tested in two different groups of patches, the mean peak current at $-100 \mathrm{mV}$ was $-255 \pm 84 \mathrm{pA}$ $(n=11)$ for the $\mathrm{SCN}^{-}{ }_{\text {in }} / \mathrm{Cl}^{-}$out condition and $-18 \pm 4 \mathrm{pA}(n=$ 7) for the gluconate ${ }^{-}{ }_{\text {in }} / \mathrm{Cl}^{-}{ }_{\text {out }}$ gradient. This is consistent with previous estimates of the contribution of anion current to transporter currents from hippocampal astrocytes in physiological chloride gradients (Table 1).

To more closely examine the relationship between coupled charge flux and the anion conductance, the kinetics of responses dominated by anion current $\left(\mathrm{SCN}^{-}{ }_{\text {in }} / \mathrm{Cl}^{-}\right.$out gradient) and by stoichiometric current (gluconate ${ }^{-}{ }_{\text {in }} / \mathrm{Cl}^{-}$out gradient) were compared. On average, the $1 / 2$-decay time in gluconate ${ }^{-}{ }_{\text {in }} / \mathrm{Cl}^{-}$out measured between -80 and $-100 \mathrm{mV}$ was $0.82 \pm 0.11 \mathrm{msec}(n=$ 7 ), whereas in the $\mathrm{SCN}^{-}{ }_{\text {in }} / \mathrm{Cl}^{-}{ }_{\text {out }}$ gradient, it was $1.07 \pm 0.06$ msec $(n=14)$. This suggests that stoichiometric current develops and subsides slightly faster than anion conductance (although not significantly; $p>0.025)$. Moreover, anion and stoichiometric 

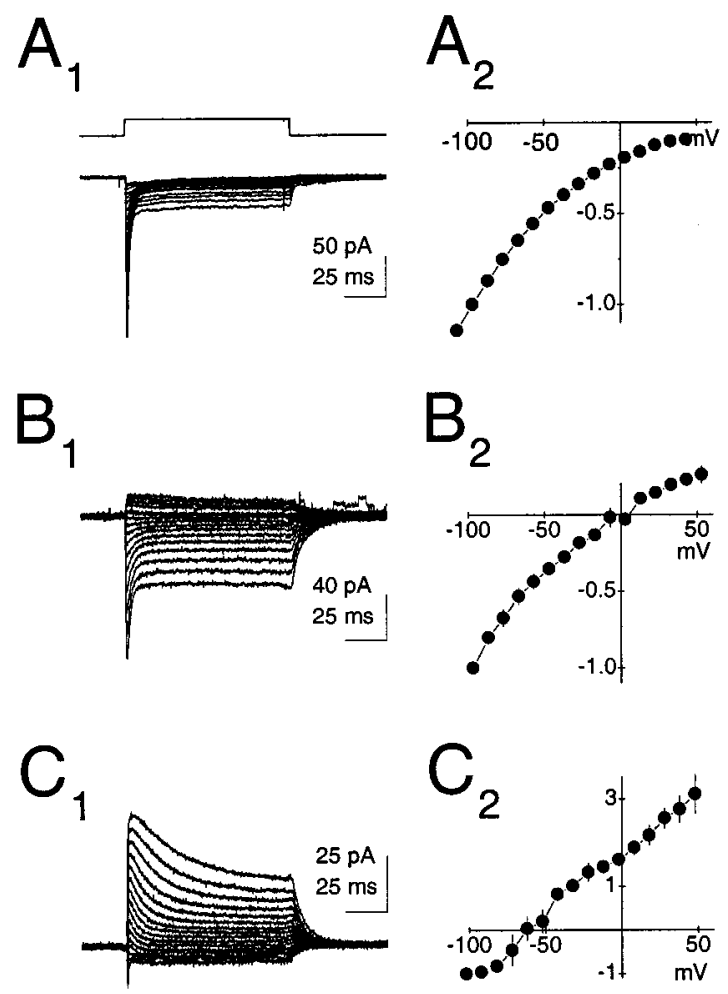

$D_{1}$

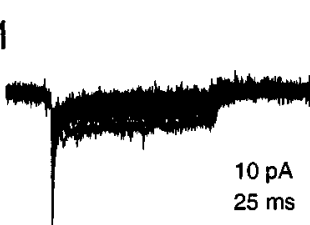

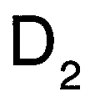

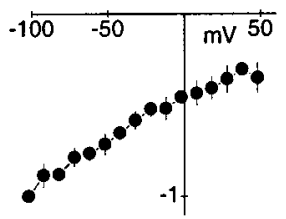

Figure 6. Different anion gradients can be used to discriminate the stoichiometric and the anion components of transporter current. $A_{1}-D_{1}$ display families of traces recorded at different membrane potentials $(-100$ to $+50 ; \Delta V, 10 \mathrm{mV})$ using the following gradients of major anions

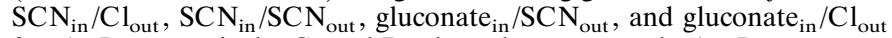
for $A-D$, respectively. $\mathrm{C}_{1}$ and $\mathrm{D}_{1}$ show the same patch. $\mathrm{A}_{2}-D_{2}$ represent mean $I-V$ relationships for these same combinations of solutions. The data were taken from $\mathrm{n}=11,4,8$, and 7 patches for $A-D$. For comparison, responses in $A$ are dominated by the anion current, and responses in $D$ are dominated by stoichiometric component of the transporter current.

components slowed in parallel at positive potentials (Fig. $\left.6 A_{1}, D_{1}\right)$, perhaps reflecting voltage dependence of the early events in the transport cycle. These results can be generally interpreted to reflect fast charge translocation steps (giving rise to the stoichiometric current) and slightly slower anion conducting steps, which are confined to a region at the beginning of a multistep cycle. This region of the cycle is rapidly and transiently occupied by most of the transporters after nearly synchronous binding. Thereafter, transporters "desynchronize" and equilibrate throughout the cycle, their desynchronous cycling giving rise to steady-state current (Bergles et al., 1997; Otis et al., 1997).

We designed a more sensitive protocol to compare the kinetics of the anion and stoichiometric currents. Each patch was exposed to two different anion gradients. In gluconate ${ }^{-}{ }_{\text {in }} / \mathrm{SCN}^{-}$out $($Fig.
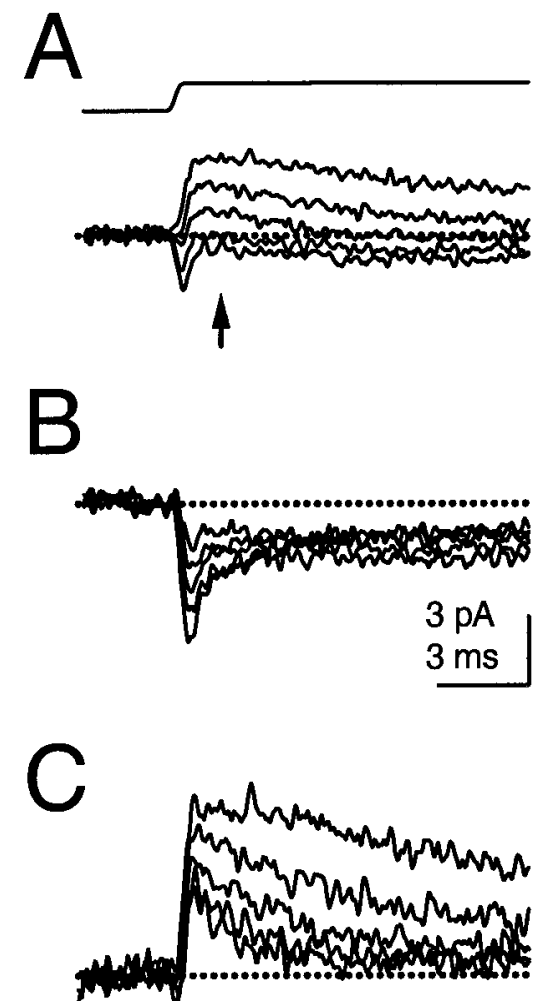

Figure 7. Stoichiometric current activates slightly faster than anion current. $A$, Patch currents at a series of membrane potentials $(-100$ to +20 ; $\Delta V, 30 \mathrm{mV}$ ) in response to $10 \mathrm{~mm}$ Glu steps with gluconate ${ }_{\text {in }} / \mathrm{SCN}_{\text {out }}$. Under these conditions, stoichiometric current is inward and anion current is outward. $B$, In the same patch and at the same holding potentials, responses with $\mathrm{Cl}_{\text {out }}$. Inward stoichiometric current dominates the small outward anion current. $C$, Subtraction of traces in $B$ from those in $A$, yielding pure anion currents carried by $\mathrm{SCN}^{-}$. Note that the outward currents are slightly slower than the inward currents. With both current components present, this is evident as an overshoot occurring after the fast inward transient (arrow in $A$ ).

$7 A$ ), both current components are apparent; inward currents represent stoichiometric current, whereas outward currents represent $\mathrm{SCN}^{-}$currents. Replacing $\mathrm{SCN}^{-}$out with $\mathrm{Cl}^{-}$out (Fig. $7 B$ ) causes the outward anion currents to disappear, leaving inward currents at all potentials. These responses are dominated by the stoichiometric component of the transporter current. Net outward current is minimal in this condition because of the relatively small contribution of anion current in physiological chloride (Arriza et al., 1994; Wadiche et al., 1995a; Levy et al., 1998). Figure $7 C$ shows a subtraction of the stoichiometric currents (Fig. 7B) from the mixed currents (Fig. 7A) yielding pure $\mathrm{SCN}^{-}$currents. From these comparisons, it is apparent that the stoichiometric current activates and deactivates slightly faster than the anion conductance. When both components are present, the slight mismatch in time course gives rise to an inward transient, followed by a delayed trough in the responses recorded at negative potentials (Fig. 7A, arrow).

Many of the measurements and conclusions in this study depend on achieving a high level of expression of the EAAT2 protein. The density of expression can be estimated from responses using gluconate ${ }^{-}$as the internal anion. The number of transporters per patch can be estimated by integrating currents over $3 \mathrm{msec}$ intervals, beginning at the Glu pulse onset, and then applying the equation $n=Q / z e_{\mathrm{o}}$, where $Q$ is the charge; $z=3$, the 
number of charges translocated in the first part of the transport cycle; and $e_{\mathrm{o}}$ is the charge per elementary particle, $1.6 \times 10^{-19} \mathrm{C}$ (Bergles and Jahr, 1997). Average charge transfer measured in this way was $29.4 \pm 6.9 \mathrm{fC}(n=7)$, yielding an estimate of $61,000 \pm 14,000$ transporters per patch. Assuming the same mean surface area for the patches as was measured in a previous study ( $\sim 7 \mu \mathrm{m}^{2}$; Bergles and Jahr, 1997), the density of transporters in our experiments is high $\left(\sim 9000 / \mu \mathrm{m}^{2}\right)$. Although this estimate is higher than the density estimated for CA1 astrocytes $(\sim 2000$ / $\mu \mathrm{m}^{2}$; Bergles and Jahr, 1997), it is similar to the density estimate of $8500 / \mu \mathrm{m}^{2}$ based on quantitative immunoblotting in hippocampus (Lehre and Danbolt, 1998). The estimate is also comparable with the density estimates for EAAT1 transporters expressed in Xenopus oocytes $\left(17,000 / \mu \mathrm{m}^{2}\right.$; Wadiche and Kavanaugh, 1998).

\section{DISCUSSION}

Recombinant EAAT2 transporter currents are similar to transporter currents in hippocampal astrocytes

EAAT2 (GLT1) is the predominant glial cell transporter in the CNS, accounting for most of the glutamate clearance in the brain (Rothstein et al., 1994, 1996; Tanaka et al., 1997). However, many astrocytic glia, such as those located in the CA1 region of the hippocampus, also express EAAT1 (GLAST) at lower levels (Rothstein et al., 1994; Lehre et al., 1995). Comparing the results in this study with those of EAAT1 (GLAST) (Wadiche and Kavanaugh, 1998) and to transporter currents from rat hippocampal astrocytes (Bergles and Jahr, 1997, 1998) supports the proposal that glutamate transport by astrocytes is dominated by EAAT2 (GLT1). Moreover, if functional transporters have a multimeric structure (Haugeto et al., 1996; Dehnes et al., 1998), these results also imply that EAAT2 operates as a homomultimer. Lastly, the data argue that the high degree of homology observed between rodent and human EAAT2 sequences (Arriza et al., 1994) dictates similar functional properties. However, these preliminary conclusions must be strengthened by studies that further examine the properties of individual transporters and combinations of coexpressed transporters.

To date, rapid solution exchange techniques have uncovered distinct kinetic properties for different transporter isoforms. Recombinant EAAT1 shows a much larger $I_{\mathrm{ss}} / I_{\text {peak }}$ ratio (0.64) and a much more slowly decaying transient ( $\tau$ of $14 \mathrm{msec}$ ) than those measured in this study (Wadiche and Kavanaugh, 1998). On Bergmann glial cells, a native transporter that is insensitive to dihydrokainate (and thus is not EAAT2/GLT1), also shows a larger $I_{\mathrm{ss}} I_{\text {peak }}$ ratio and slower transients (Bergles et al., 1997). Finally, Purkinje neuron glutamate transporters exhibit a smaller $I_{\text {ss }} / I_{\text {peak }}$ ratio $(0.14)$ and a more slowly decaying transient ( $\tau$ of 8 msec) (Otis and Jahr, 1998).

\section{A simple kinetic model can explain both components of the transporter current}

All glutamate transporters tested to date show a transient followed by a steady-state current in response to rapid applications of Glu. Although previous measurements concentrated on anion currents, in CA1 astrocytes, small stoichiometric currents were described (Bergles and Jahr, 1997). The high level of expression in this study allows a direct comparison of both components of the transporter current. To test hypotheses about how charge translocation is related to the anion conductance, we used a simple model of the transport cycle. The cycle was divided into three collections of states as shown in Figure 8: nonconducting states with substrate binding sites facing the extracellular space,

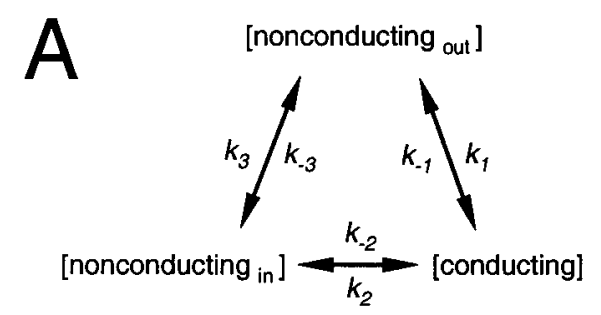

B

simulated stoichiometric current
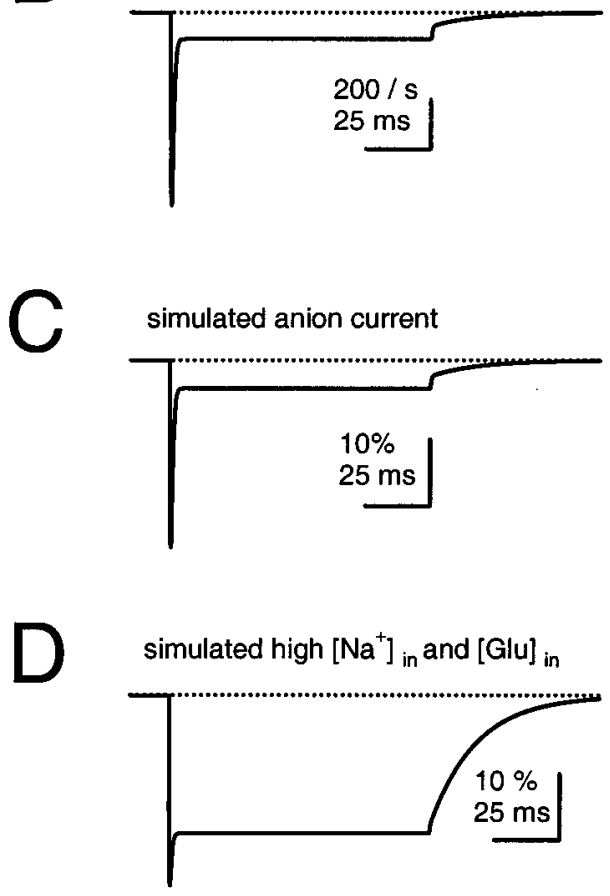

Figure 8. A simple kinetic model that accounts for the two components of transporter current. $A$, The model consists of three sets of states connected by the following rate constants: $k_{1}=0$ or $2000 / \mathrm{sec} ; k_{-1}=4 / \mathrm{sec}$; $k_{2}=480 / \mathrm{sec}^{*} \exp [(-V F) /(2 R T)] ; k_{-2}=400 / \mathrm{sec} * \exp [(V F) /(2 R T)] ;$ $k_{3}=10 / \mathrm{sec}^{*} \exp [(-V F) /(2 R T)]$; and $k_{-3}=1 / \mathrm{sec}^{*} \exp [(V F) /(2 R T)] . V$ of $-90 \mathrm{mV}$ for simulations in $B$ and $C$ and $-100 \mathrm{mV}$ in $D$. $B$, Simulated stoichiometric current (the sum of the net fluxes for the two voltagedependent transitions [conducting] $\rightarrow$ [nonconducting ${ }_{i n}$ ] and [nonconducting $\left._{\text {in }}\right] \rightarrow$ [nonconducting out $_{\text {I }}$ ) in response to a $100 \mathrm{msec}$ duration jump in the value of $k_{1}$ from 0 to $2000 / \mathrm{sec}$ (to simulate the Glu pulse). $C$, Simulated anion current (the occupancy of [conducting]) in response to the Glu pulse. $D$, Anion current in response to the Glu pulse with the effects of high $[\mathrm{Na}]_{\text {in }}$ and $[\mathrm{Glu}]_{\text {in }}$ simulated by increasing the rate constant factor in $k_{-2}$ from $400 / \mathrm{sec}$ to $6000 / \mathrm{sec}$.

designated [nonconducting ${ }_{\text {out }}$; anion conducting states, designated [conducting]; and nonconducting states with substrate binding sites facing the intracellular space, designated [nonconducting $\left._{\text {in }}\right]$. In the model, conducting transporters are formed after the binding of substrate and cotransported ions $\left(\mathrm{Na}^{+}\right.$and $\left.\mathrm{H}^{+}\right)$. The small conductance seen in the absence of substrate (shown in Fig. $2 B, C$ ) has been omitted for simplicity. As an additional simplification, individual binding steps are not explicit in the model (none of the rate constants are concentration-dependent). Forward rate constants (i.e., those leading to Glu uptake) are denoted by positive subscripts, whereas backward rate constants have negative subscripts. 
Two forward and two backward rate constants have voltage dependence of the form

$$
\begin{aligned}
k_{\text {forward }}=k_{\mathrm{f} 0} \exp [-(z V F) /(2 R T)] \quad \text { and } & k_{\text {backward }} \\
= & k_{\mathrm{b} 0} \exp [(z V F) /(2 R T)],
\end{aligned}
$$

where $k_{\mathrm{fo}}$ and $k_{\mathrm{b} 0}$ are the forward and backward rate constants at $0 \mathrm{mV} ; z=1$, the valence of the charge movement; $V$ is the membrane potential; $T=298-K$; and $F$ and $R$ are Faraday's constant and the gas constant, respectively. Charge movements were assumed to traverse symmetric energy barriers and to cross the entire field (Läuger, 1991). Reflecting the established stoichiometry for the transport cycle $\left(1 \mathrm{Glu}^{-} / 3 \mathrm{Na}^{+} / 1 \mathrm{H}^{+}\right.$cotransported and $1 \mathrm{~K}^{+}$counter-transported) (Zerangue and $\mathrm{Ka}$ vanaugh, 1996a; Levy et al., 1998), the total charge movement per cycle (i.e., the sum of $z$ values for a complete cycle) was constrained to two. Stoichiometric current was simulated by summing the net charge flux that occurs as a result of the two voltagedependent steps, [conducting] $\rightarrow$ [nonconducting $_{\text {in }}$ ] and [nonconducting $\left._{\text {in }}\right] \rightarrow$ [nonconducting $\left.{ }_{\text {out }}\right]$.

Simulations of the anion current and stoichiometric charge flux in response to a [Glu] jump are shown in Figure 8. Many features of both components of transporter current are captured by the model. These include a rapid rise-to-peak, a quickly decaying transient $(1 / 2$-decay times for stoichiometric and anion currents is $0.8 \mathrm{msec}$ ), a double-exponential deactivation, and a steady-state level of $\sim 15 \%$ of the peak. Simulated effects of high $\left[\mathrm{Na}^{+}\right]_{\text {in }}$ and $[\mathrm{Glu}]_{\text {in }}\left(I_{\mathrm{ss}} / I_{\text {peak }}, 0.72\right.$ at $\left.-100 \mathrm{mV}\right)($ Fig. $8 D)$ are similar to the data. Although not shown, the model also reproduces other behaviors of the transporter such as the rate of recovery ( $\tau$ of 17.4 msec) and the slowing of the transient at positive membrane potentials.

Several general insights can be drawn from this simple model. First, the model provides an explanation for why large transient and smaller steady-state responses are observed for both the anion and stoichiometric components of the transporter current. This results from movement through a multistate cycle in which only a subset of states are experimentally detectable (the [conducting] states). To generate the transient, movement through these states must be rapid and synchronous. This is accomplished by trapping transporters in the absence of Glu in the [nonconducting $\left._{\text {out }}\right]$ states. Glu binding can then trigger nearly synchronous entry into the [conducting] collection of states. Transporters eventually become desynchronized relative to one another because, in the presence of Glu, they have access to all states in the cycle.

A second prediction is that the steps generating stoichiometric and anion components of the current occur at very similar points in the transport cycle. This provides support for physical models in which the transporter protein adopts a configuration that is leaky to anions during the translocation of glutamate and cotransported ions (Wadiche et al., 1995a).

Last, the model explains why the relative contribution of the steady-state anion current is larger under exchange conditions (Billups et al., 1996; Otis and Jahr, 1998). Simulations of high [Glu] and $\left[\mathrm{Na}^{+}\right]$on both sides of the membrane confines transporters to the [conducting] collection of states, making it unlikely that transporters remain unbound or enter other states in the cycle.

This is a simplified version of previous models that we and others have developed (Billups et al., 1996; Larsson et al., 1996; Otis and Jahr, 1998; Wadiche and Kavanaugh, 1998). Although the present model fails to describe all aspects of the data, including the conductance observed in the absence of substrate (Fig. 2) and the concentration dependencies of transitions, the simplifications introduced result in fewer free parameters and more easily understood dynamic behavior. More detailed models that explicitly incorporate substrate and ion binding are essential for a complete description of the transporter.

\section{Different deactivation pathways suggest that transporters have high efficiency}

Efficient glutamate transport requires Glu binding in the extracellular space, dissociation into the intracellular compartment, and recycling of the empty binding site. In contrast, in ionic gradients favoring exchange, complete cycles of transport are believed to be extremely rare. In the exchange mode, transporters bind Glu, activate an anion conducting state, and deactivate by unbinding Glu into the extracellular space. Independent experimental evidence supporting the existence of exchange modes of operation has been provided by biochemical measurements of substrate and ion flux (Kanner and Sharon, 1978; Kanner and Bendahan, 1982; Kavanaugh et al., 1997; Zhang et al., 1998).

Different fates for Glu under conditions of high internal $\mathrm{Na}^{+} /$ Glu (exchange) or 0 internal $\mathrm{Na}^{+} / \mathrm{Glu}$ are reflected in different time courses of deactivation and recovery of the anion conductance (Figs. 4, 5). Under exchange conditions, both deactivation and recovery must occur by reversal and unbinding to the extracellular space. Glu pulses with 0 internal $\mathrm{Na}^{+}$elicit faster, voltage-independent deactivation kinetics, suggesting that forward transitions away from anion conducting states rapidly sequester transmitter and thereby prevent the voltage-dependent route of reversal and unbinding. This dichotomy in deactivation behavior supports the proposal that EAAT2 efficiently translocates Glu under physiological conditions. This may not be the case for all transporter subtypes; significantly different kinetics have been observed in concentration jump experiments on other glutamate transporter subtypes (Wadiche and Kavanaugh, 1998; A. Tzingounis and M. P. Kavanaugh, unpublished observations). In contrast to EAAT2, kinetic modeling of EAAT1/GLAST suggests that it is more probable for glutamate to unbind to the outside than to be transported (Wadiche and Kavanaugh, 1998).

The importance of translocation efficiency is highlighted by recent experiments on hippocampal neurons cocultured with glia (Mennerick et al., 1999). Glial cell depolarization was shown to slow excitatory synaptic currents. However, depolarization did not affect glutamate affinity for the transporter, implying that depolarization prolongs the lifetime of glutamate without reducing binding but by slowing the translocation of glutamate after binding. These results argue that key steps after binding, perhaps encompassing translocation of substrate and ions, are critical for determining the functional properties of glutamate transporters. The present data are consistent with rapid and efficient sequestration of glutamate by EAAT2 during synaptic transmission. Furthermore, the data provide strong confirmation that anion conductance is tightly associated with the transport cycle and that it can be used to estimate glutamate transport in situ.

\section{REFERENCES}

Arriza JL, Fairman WA, Wadiche JI, Murdoch GH, Kavanaugh MP, Amara SG (1994) Functional comparisons of three glutamate transporter subtypes cloned from human motor cortex. J Neurosci 14:5559-5569.

Arriza JL, Eliasof S, Kavanaugh MP, Amara SG (1997) Excitatory amino acid transporter 5, a retinal glutamate transporter coupled to a chloride conductance. Proc Natl Acad Sci USA 94:4155-4160. 
Bergles DE, Jahr CE (1997) Synaptic activation of glutamate transporters in hippocampal astrocytes. Neuron 19:1297-1308.

Bergles DE, Jahr CE (1998) Glial contribution to glutamate uptake at Schaffer collateral-commissural synapses in the hippocampus. J Neurosci 18:7709-7716.

Bergles DE, Dzubay JA, Jahr CE (1997) Glutamate transporter currents in Bergmann glial cells follow the time course of extrasynaptic glutamate. Proc Natl Acad Sci USA 94:14821-14825.

Billups B, Rossi D, Attwell D (1996) Anion conductance behavior of the glutamate uptake carrier in salamander retinal glial cells. J Neurosci 16:6722-6731.

Dehnes Y, Chaudhry FA, Ullensvang K, Lehre KP, Storm-Mathisen J, Danbolt NC (1998) The glutamate transporter EAAT4 in rat cerebellar Purkinje cells: a glutamate-gated chloride channel concentrated near the synapse in parts of the dendritic membrane facing astroglia. J Neurosci 18:3606-3619.

Diamond JD, Jahr CE (1997) Transporters buffer synaptically released glutamate on a millisecond time scale. J Neurosci 17:4672-4687.

Erecinska M, Wantorsky D, Wilson DF (1983) Aspartate transport in synaptosomes from rat brain. J Biol Chem 258:9069-9077.

Fairman WA, Vandenberg RJ, Arriza JL, Kavanaugh MP, Amara SG (1995) An excitatory amino-acid transporter with properties of a ligand-gated chloride channel. Nature 375:599-603.

Haugeto O, Ullensvang K, Levy LM, Chaudhry FA, Honore T, Nielsen M, Lehre KP, Danbolt NC (1996) Brain glutamate transporter proteins form homomultimers. J Biol Chem 271:27715-27722.

Kanai Y, Hediger MA (1992) Primary structure and functional characterization of a high-affinity glutamate transporter. Nature 360:467-471.

Kanner BI, Bendahan A (1982) Binding order of substrates to the sodium and potassium ion coupled L-glutamic acid transporter from rat brain. Biochemistry 21:6327-6330.

Kanner BI, Sharon I (1978) Active transport of L-glutamate by membrane vesicles isolated from rat brain. Biochemistry 17:3949-3953.

Kavanaugh MP, Bendahan A, Zerangue N, Zhang Y, Kanner BI (1997) Mutation of an amino acid residue influencing potassium coupling in the glutamate transporter GLT-1 induces obligate exchange. J Biol Chem 272:1703-1708.

Larsson HP, Picaud SA, Werblin FS, Lecar H (1996) Noise analysis of the glutamate-activated current in photoreceptors. Biophys $\mathrm{J}$ 70:733-742.

Läuger P (1991) Electrogenic ion pumps. Sunderland, MA. Sinauer.

Lehre KP, Danbolt NC (1998) The number of glutamate transporter subtype molecules at glutamatergic synapses: chemical and stereological quantification in young adult rat brain. J Neurosci 18:8751-8757.

Lehre KP, Levy LM, Ottersen OP, Storm-Mathisen J, Danbolt NC (1995) Differential expression of two glial glutamate transporters in the rat brain: quantitative and immunocytochemical observations. J Neurosci 15:1835-1853.
Levy LM, Warr O, Attwell D (1998) Stoichiometry of the glial glutamate transporter GLT-1 expressed inducibly in a Chinese hamster ovary cell line selected for low endogenous $\mathrm{Na}^{+}$-dependent glutamate uptake. J Neurosci 18:9620-9628.

Mennerick S, Shen W, Xu W, Benz A, Tanaka K, Shimamoto K, Isenberg KE, Krause JE, Zorumski CF (1999) Substrate turnover by transporters curtails synaptic glutamate transients. J Neurosci 19:9242-9251.

Otis TS, Jahr CE (1998) Anion currents and predicted glutamate flux through a neuronal glutamate transporter. J Neurosci 18:7099-7110.

Otis TS, Kavanaugh MP, Jahr CE (1997) Postsynaptic glutamate transport at the climbing fiber-Purkinje cell synapse. Science 277:1515-1518.

Pines G, Danbolt NC, Bjoras M, Zhang Y, Bendahan A, Eide L, Koepsell H, Storm-Mathisen J, Seeberg E, Kanner BI (1992) Cloning and expression of a rat brain L-glutamate transporter. Nature 360:464-467.

Rothstein JD, Dykes-Hoberg M, Pardo CA, Bristol LA, Jin L, Kuncl RW, Kanai Y, Hediger MA, Wang Y, Schielke JP, Welty DF (1996) Knockout of glutamate transporters reveals a major role for astroglial transport in excitotoxicity and clearance of glutamate. Neuron 16:675-686.

Rothstein JD, Martin L, Levey AI, Dykes-Hoberg M, Jin L, Wu D, Nash N, Kuncl RW (1994) Localization of neuronal and glial glutamate transporters. Neuron 13:713-725.

Stallcup WB, Bulloch K, Baetge EE (1979) Coupled transport of glutamate and sodium in a cerebellar nerve cell line. J Neurochem 32:57-65.

Storck T, Schulte S, Hofmann K, Stoffel W (1992) Structure, expression, and functional analysis of a $\mathrm{Na}\left({ }^{+}\right)$-dependent glutamate/aspartate transporter from rat brain. Proc Natl Acad Sci USA 89:10955-10959.

Tanaka K, Watase K, Manabe T, Yamada K, Watanabe M, Takahashi K, Iwama H, Nishikawa T, Ichihara N, Kikuchi T, Okuyama S, Kawashima N, Hori S, Takimoto M, Wada K (1997) Epilepsy and exacerbation of brain injury in mice lacking the glutamate transporter GLT-1. Science 276:1699-1702.

Wadiche JI, Kavanaugh MP (1998) Macroscopic and microscopic properties of a cloned glutamate transporter/chloride channel. J Neurosci 18:7650-7661.

Wadiche JI, Amara SG, Kavanaugh MP (1995a) Ion fluxes associated with excitatory amino acid transport. Neuron 15:721-728.

Wadiche JI, Arriza JL, Amara SG, Kavanaugh MP (1995b) Kinetics of a human glutamate transporter. Neuron 14:1019-1027.

Zerangue N, Kavanaugh MP (1996a) Flux coupling in a neuronal glutamate transporter. Nature 383:634-637.

Zerangue N, Kavanaugh MP (1996b) Interaction of L-cysteine with a human excitatory amino acid transporter. J Physiol (Lond) 493:419-423.

Zhang Y, Bendahan A, Zarbiv R, Kavanaugh MP, Kanner BI (1998) Molecular determinant of ion selectivity of a $\left(\mathrm{Na}^{+}+\mathrm{K}^{+}\right)$-coupled rat brain glutamate transporter. Proc Natl Acad Sci USA 95:751-755. 